\title{
Esófago de Barrett, diagnóstico y vigilancia
}

\author{
Francisco Valdovinos-Andraca* y Héctor A. Díaz-Hernández \\ Instituto Nacional de Ciencias Médicas y Nutrición Salvador Zubirán, Ciudad de México, México
}

\section{Resumen}

En las reuniones anuales internacionales de la unión europea del 2019 y de los Estados Unidos de América (E.U.A.) del 2020 se presentaron diversos trabajos de gran interés sobre el tamizaje, diagnóstico y vigilancia esófago de Barrett. Dentro de los más destacados se encuentran estudios sobre la técnica de muestreo amplio transepitelial y análisis histológico tridimensional asistido por computadora tanto en el diagnóstico como en la vigilancia del esófago de Barrett, otros estudios en la evaluación de técnicas de inteligencia artificial en la detección de displasia/neoplasia en esófago de Barrett, entre otros de actualidad.

Palabras clave: Esófago de Barrett. Diagnóstico. Vigilancia. Displasia. Adenocarcinoma de esófago.

\section{Tamizaje y diagnóstico del esófago de Barrett}

En un estudio retrospectivo en E.U.A., se observó que el realizar una esofagogastroduodenoscopia, como estudio adicional al realizar una colonoscopia de tamizaje, puede detectar un número importante de lesiones pre-neoplásicas y neoplásicas en esófago y estómago, principalmente en regiones de riesgo intermedio a alto, por lo cual, se sugiere ampliar con estudios prospectivos esta alternativa ${ }^{1}$. Por otro lado, unos estudios en Singapur y Japón, encontraron que pacientes con constipación crónica y neoplasias en colon, tienen mayor riesgo de presentar esófago de Barrett (EB), por lo cual, se propone considerar el estudio de estas condiciones como potenciales factores de riesgo para $\mathrm{EB}^{2,3}$.

En estudios en centros individuales y multicéntricos se observó que la técnica de muestreo amplio trans-epitelial y análisis histológico tridimensional asistido por computadora, al usarse de manera adicional a la toma de biopsias endoscópicas de áreas con sospecha de esófago de Barrett, incrementa el rendimiento diagnóstico de esta patología, por lo que se sugiere que pudiera ser una técnica adyuvante en el diagnóstico del EB ${ }^{4,5}$.

Actualmente, la presencia de una línea $Z$ irregular 0 un esófago de Barrett de segmento ultra-corto se consideran condiciones de muy bajo riesgo de progresión a displasia y neoplasia, por lo cual, no se les suele dar seguimiento. Sin embargo, en el estudio de Kandasamy et al. se observó que en ciertos sub-grupos de pacientes con estas condiciones y factores de riesgo como edad avanzada, género masculino, raza caucásica, obesidad, consumo de tabaco y alcohol, existe una incidencia importante de displasia y neoplasia, por lo cual, se propone estudiar más a fondo la necesidad de dar seguimiento con vigilancia endoscópica a estos grupos de pacientes 6 .

\section{Vigilancia del esófago del Barrett}

En un estudio multicéntrico en los EE.UU. se evaluó de manera prospectiva el comportamiento de la

\section{Correspondencia:}


incidencia de displasia/neoplasia en cuanto a su distribución circunferencial en la superficie del esófago utilizando endoscopia de alta definición con luz blanca y endomicroscopia láser volumétrica, se observó, que la distribución de la incidencia en los cuatro cuadrantes es uniforme, a diferencia de lo reportado previamente en estudios retrospectivos, por lo cual, se concluye que la vigilancia en el esófago de Barrett debe ser igualmente minuciosa en los cuatro cuadrantes de la luz esofágica?.

En un estudio multicéntrico en Europa se demostró que la técnica de muestreo amplio transepitelial y análisis histológico tridimensional asistido por computadora, sumada a la toma de biopsias habitual con el protocolo de Seattle, aumenta el rendimiento en la detección de displasia y neoplasia en pacientes con $\mathrm{EB}$, obteniendo con la combinación de ambas técnicas, una tasa de detección para displasia y neoplasia de

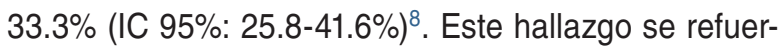
za también por un metaanálisis en el que dicha técnica agregada al protocolo de Seattle incrementó el rendimiento en la detección de displasia y neoplasia a un 34.5\% (IC 95\%: 8-76\%) con un incremento absoluto del 5\% (IC 95\%: 2-7\%) $)^{4,5}$. Este aumento en la detección de displasia es independiente de la longitud del EB ${ }^{9}$.

En estudios en los E.U.A. y Reino Unido se ha creado y evaluado un programa de inteligencia artificial basado en redes neuronales convolucionales y se ha aplicado en tiempo real a videos de pacientes con esófago de Barrett en la detección de displasia y neoplasia, logrando una sensibilidad del $91.0-96.7 \%$, especificidad del $87.6-96.0 \%$ y precisión diagnóstica del $93.7 \%{ }^{10-12}$. Por otro lado, en un estudio experimental en los Países Bajos se probó un algoritmo computacional en tiempo real para la detección de neoplasia en pacientes con esófago de Barrett, logrando una sensibilidad del $100 \%$, especificidad del $90 \%$ y precisión diagnóstica del $90 \%{ }^{13}$. Groof et al. también validó esta técnica interna y externamente con una precisión diagnóstica del $88 \%{ }^{14}$. En modelos de inteligencia artificial para estos fines se ha propuesto que los modelos basados en pixeles pueden ser más aptos para su uso en tiempo-real ${ }^{15}$. Si bien estos han sido estudios en muestras pequeñas, estos resultados, motivarán el realizar estudios prospectivos a mayor escala para evaluar esta técnica de mejor manera.

La displasia de bajo grado continúa siendo sobre-diagnosticada por médicos patólogos no expertos, un estudio mostró que un inmunoensayo es de utilidad en identificar casos de displasia de bajo grado, el cual, pudiera ser una técnica útil para la estandarización del diagnóstico de esta condición ${ }^{16}$. Por otro lado, se presentaron estudios sobre la historia natural de la displasia de bajo grado que reportaron tasas del 10\% de progresión anual a displasia de alto grado o adenocarcinoma, y en otros, una media de 10 meses para progresión a displasia de alto grado o adenocarcinoma, apoyando, la conducta de considerar el tratamiento temprano de ablación en este contexto ${ }^{17}$.

\section{Vigilancia de recurrencia de displasia/ neoplasia tratada del esófago del Barrett}

En un metaanálisis en los EE.UU. se observó que la técnica de muestreo amplio trans-epitelial y análisis histológico tridimensional por computadora, de manera adicional a la toma de biopsias endoscópicas en el seguimiento de pacientes con esófago de Barrett tratado, mejora la detección de persistencia y recurrencia de metaplasia intestinal ${ }^{18}$. Suresh Kumar et al. observaron que por medio de endomicroscopia láser volumétrica, la ausencia de glándulas epiteliales a nivel de unión esófago-gástrica correlaciona con la erradicación completa de la metaplasia intestinal, por lo cual, se propone que podría ser una técnica auxiliar en el seguimiento posterior al tratamiento ablativo en pacientes con $\mathrm{EB}^{19}$.

En un estudio multicéntrico en los Estados Unidos de América se evaluó el intervalo óptimo para la vigilancia de recurrencia de displasia/neoplasia tratada en pacientes con esófago de Barrett, y se determinó que la estrategia adecuada de vigilancia, es la revisión endoscópica cada 6 meses por 2 años, y cada año en adelante $^{20}$. Además, Um et al. observaron que la adherencia de los pacientes al seguimiento cada 3 meses en el primer año, es tan baja como del $30.7 \% 2^{1}$. Con estos resultados, es posible que a futuro cambie la tendencia del seguimiento en el primer año de la erradicación completa de la metaplasia intestinal en los pacientes con esófago de Barrett tratado, y en lugar de ser cada 3 meses, sea cada 6 meses durante el primer año.

\section{Conclusiones}

En los últimos años se han documentado avances en el diagnóstico del EB y en la detección de displasia/ neoplasia adicionando a la toma de biopsias convencional, técnicas especiales de muestreo.

Se confirma que la displasia de bajo grado debe diagnosticarse de manera precisa por su riesgo de progresión a displasia de alto grado. 
Se está evaluando a nivel mundial la aplicabilidad de la inteligencia artificial en la detección de displasia/ neoplasia en EB.

Los lineamientos de la vigilancia del EB se han extendido a aquellos pacientes que ya han recibido tratamiento endoscópico y que debemos conocer cómo y cuándo vigilarlos.

\section{Financiamiento}

Los autores no recibieron patrocinio para llevar a cabo este artículo.

\section{Conflicto de intereses}

Los autores declaran no tener conflicto de intereses alguno.

\section{Bibliografía}

1. Lau WLJ, Khoo MJW, Leong XH, et al. Opportunistic detection of oesophagogastric neoplastic and pre-neoplastic lesions during screening colonoscopy program - a worthwhile strategy? [Internet]. UEG Week Abstract Book 2019. Available from: https://ueg.eu/files/778/ e07413354875be01a996dc560274708e.pdf

2. Asfari MM, Sarmini MT, Ellison SB, et al. Assessing the risk for gastroesophageal reflux disease and its complications in patients with constipation. [Internet]. UEG Week Abstract Book 2019. Available from: https:// ueg.eu/files/778/e07413354875be01a996dc560274708e.pdf

3. Nakahara R, Yuki T, Ujihara T, et al. Relationship between Barrett's esophagus and colonic diseases: Reference to endoscopic surveillance. [Internet]. UEG Week Abstract Book 2019. Available from: https://ueg.eu/ files/778/e07413354875be01a996dc560274708e.pdf

4. Chandan S, Mashiana $H$, Mohan $B$, et al. Clinical applicability of wide area transepithelial sampling (WATS-3D) in screening \& surveillance of Barrett's esophagus - a systematic review \& sensitivity meta-analysis. [Internet]. DDW ePosters. 2020. Available from: https://ddw.apprisor.org/ epsSearchDDW.cfm

5. Suresh-Kumar VC, Subedi A, Suresh-Patthipati V, et al. Wide-area transepithelial sampling in adjunct to forceps biopsy increases the absolute detections rates of barrett's esophagus and esophageal dysplasia: a metaanalysis and systematic review. [Internet]. DDW ePosters. 2020. Available from: https://ddw.apprisor.org/epsSearchDDW.cfm

6. Kandasamy C, Bilal M, Freedman B, et al. Clinical characteristics associated with dysplasia and esophageal adenocarcinoma in patients with an irregular z-line. [Internet]. DDW ePosters. 2020. Available from: https://ddw.apprisor.org/epsSearchDDW.cfm

7. Martínez N, Raphael K, Cavaliere K, et al. Clock-face distribution of visible vs invisible dysplasia in barrett's esophagus: a pooled analysis of three prospective studies. [Internet]. DDW ePosters. 2020. Available from: https://ddw.apprisor.org/epsSearchDDW.cfm
8. Bisschops R, Haidry R, Messmann $\mathrm{H}$, et al. Wide area transepithelial sample esophageal biopsy combined with computer assisted 3-dimensional tissue analysis (WATS3D) for detection of high grade dysplasia and adenocarcinoma in barrett: european multi-center, prospective, randomized, tandem study. [Internet]. DDW ePosters. 2020. Available from: https://ddw.apprisor.org/epsSearchDDW.cfm

9. Lightdale C, Trindade A, Judath J, et al. Dysplasia detection rates are independent of barrett's esophagus segment length when using wide area transepithelial sampling. [Internet]. DDW ePosters. 2020. Available from: https://ddw.apprisor.org/epsSearchDDW.cfm

10. Hussein M, Gonzalez-Bueno Puyal J, Brandao P, et al. deep neural network for the detection of early neoplasia in barrett's oesophagus. [Internet]. DDW ePosters. 2020. Available from: https://ddw.apprisor.org/epsSearchDDW.cfm

11. Choi A, Hashimoto R, Cavaliere $K$, et al. High accurancy and effectiveness with deep neural networks and artificial intelligence in detection of early esophageal neoplasia in barrett's esophagus: an external video validation study. [Internet]. DDW ePosters. 2020. Available from: https:// ddw.apprisor.org/epsSearchDDW.cfm

12. Hashimoto R.1, El Hage Chehade N.2, Dao T.1, et al. high accuracy and effectiveness with deep neural networks and artificial intelligence in detection of early esophageal neoplasia in barrett's esophagus [Internet]. UEG Week Abstract Book 2019. Available from: https://ueg.eu/files/778/ e07413354875be01a996dc560274708e.pdf

13. Struyvenberg M, Groof J, Fockens K, et al. Computer-aided detection algorithm detects Barrett neoplasia with high diagnostic accuracy during live endoscopic procedures: a pilot study. [Internet]. DDW ePosters. 2020. Available from: https://ddw.apprisor.org/epsSearchDDW.cfm

14. Groof J, Struyvenberg M, van der Sommen F, et al. Deep learning system detects barrett's esophagus neoplasia with high accuracy in a multi-step training and external validation study. [Internet]. UEG Week Abstract Book 2019. Available from: https://ueg.eu/files/778/e07413354875be01a996dc560274708e.pdf

15. Hussein M, Saiko M, Masaike Y, et al. Pixel vs patch-based deep learning models, paving the way towards real-time computerassissted detection of barrett's neoplasia. [Internet]. DDW ePosters. 2020. Available from: https://ddw.apprisor.org/epsSearchDDW.cfm

16. Frei N, Konté K, Bossart E, et al. An automated, quantitative multiplex immunofluorescence assay accurately risk stratifies Barrett's esophagus patients with a community-based diagnosis of low -grade dyspl asia at a rate comparable to expert pathologists. [Internet]. UEG Week Abstract Book 2019. Available from: https://ueg.eu/files/778/e07413354875be01a996dc560274708e.pdf

17. Hussein M, Sehgal V, Magee C, et al. The natural history of low-grade dysplasia in patients with Barrett's Oesophagus: A tertiary centre experience. [Internet]. UEG Week Abstract Book 2019. Available from: https:// ueg.eu/files/778/e07413354875be01a996dc560274708e.pdf

18. Suresh Kumar VC, Subedi A, Suresh Patthipati V, et al. Wide-area transepithelial sampling in adjunct to forceps biopsy increases the diagnostic yield of recurrent or residual barrett's esophagus in patients who underwent endoscopic treatment: a meta-analysis and systematic review. [Internet]. DDW ePosters. 2020. Available from: https://ddw.apprisor.org/epsSearchDDW.cfm

19. Yarlagadda M, Kamboj A, Kahn A, et al. Epithelial gland burden determined by volumetric laser endomicroscopy is associated with complete remission of intestinal metaplasia following endoscopic eradication therapy for Barrett's esophagus. [Internet]. DDW ePosters. 2020. Available from: https://ddw.apprisor.org/epsSearchDDW.cfm

20. Kahn A, Crook J, Sami S, et al. Optimization of surveillance intervals after successful radiofrequency ablation therapy for dysplastic Barrett's esophagus: results of an international multicenter cohort study. [Internet]. DDW ePosters. 2020. Available from: $h$ ttps://ddw. apprisor.org/epsSearchDDW.cfm

21. Um $P$, Chan $M$, Hinton $A$, et al. Adherence to recommended surveillance intervals is challenging for Barrett's esophagus patients with high-grade dysplasia and carcinoma in-situ. [Internet]. DDW ePosters. 2020. Available from: https://ddw.apprisor.org/epsSearchDDW.cfm 